\title{
Increase of bone marrow lymphocytes in systemic mastocytosis: reactive lymphocytosis or malignant lymphoma? Immunohistochemical and molecular findings on routinely processed bone marrow biopsy specimens
}

\author{
H-P Horny, K Lange, K Sotlar, P Valent
}

J Clin Pathol 2003;56:575-578

See end of article for authors' affiliations

......................

Correspondence to Dr H-P Horny, Ratzeburger Allee 160, Institute of Pathology, University of Lübeck,

D-23538 lübeck,

Germany;

horny@patho.mu-luebeck.de

Accepted for publication 14 March 2003

\begin{abstract}
Aims: To clarify the nature (reactive or neoplastic) of lesional, perifocally aggregated lymphocytes in bone marrow infiltrates of systemic mastocytosis (SM), the histopathology of which can resemble malignant lymphoma with focal bone marrow involvement, particularly low grade malignant B cell lymphoma of lymphoplasmacytic immunocytoma subtype, which frequently exhibits increased mast cell (MC) numbers.

Methods: Thirteen cases of SM and three of lymphoplasmacytic immunocytoma with predominant focal bone marrow infiltration were investigated. Immunostaining of formalin fixed, paraffin wax embedded bone marrow specimens was performed using antibodies against CD2, CD5, CD20, CD23, and CD25; $\kappa$ and $\lambda$ immunoglobulin light chains; and MC markers chymase, tryptase, and CD 117 (KIT). Monoclonal rearrangements of $\operatorname{lgH}$ and TCR $\gamma$ were studied using seminested polymerase chain reaction (PCR). c-kit point mutation Asp8 16-Val was detected by PNA mediated PCR clamping and probes.

hybridisation

Results: The lymphocytic clusters in SM contained nearly equal numbers of mature $T$ and $B$ cells, the latter with no coexpression of aberrant antigens, such as CD5 or CD23. Most MCs in SM cases constantly coexpressed tryptase, CD25, and CD117. No monoclonal rearrangements were seen for $\lg H$ or TCR $\gamma$. In contrast, B cells from immunocytomas showed light chain restriction and monoclonal rearrangement for lgH, confirming their neoplastic nature. c-kit point mutation Asp8 16-Val was found in ten of $13 \mathrm{SM}$ cases, but in none of the three immunocytomas.

Conclusions: Focal accumulations of lymphocytes in the bone marrow of SM are reactive in nature and could be termed lymphocytosis. A diagnosis of SM-AHNMD/immunocytoma should not be made.
\end{abstract}

\footnotetext{
"To make the diagnosis of systemic mastocytosis, bone marrow histology must show, by definition, at least one compact or dense mast cell infiltrate"
}

The following questions were investigated by our combined immunohistochemical and molecular biological study of routinely processed bone marrow biopsy specimens: (1) Are lymphocytic aggregates (and diffusely scattered lymphocytes) in the bone marrow of patients with indolent SM benign or neoplastic? (2) Do cases exist with concomitant occurrence of mastocytosis and low grade non-Hodgkin lymphoma (NHL), that should be designated SM-NHL according to the new system of classification of mastocytosis? (3) How useful are the diagnostic criteria included in the recently published revised system of classification of mastocytosis when only bone marrow trephine biopsy specimens are available?

\section{METHODS}

In total, 16 routinely processed bone marrow biopsy specimens were analysed. All tissue specimens had been fixed in $5 \%$ buffered formalin, embedded in paraffin wax, and mildly decalcified in edetic acid for six to eight hours. In addition to conventional staining, such as Giemsa, naphthol AS-D chloroacetate esterase, and Gömöri's silver impregnation, sections were immunostained using the avidin-biotin complex method with antibodies against various lymphocyte and MC

Abbreviations: $M C$, mast cell; $N H L$, non-Hodgkin lymphoma; PCR, polymerase chain reaction; SM, systemic mastocytosis 
Table 1 Diagnosis, presence of lymphocytic clusters (LC), monoclonality of $\operatorname{lgH} / \mathrm{TCR} \gamma$, and point mutation Asp8 16-Val of c-kit

\begin{tabular}{llllll}
\hline Case & Diagnosis & LC & IgH & TCR & c-kit \\
\hline 1 & Indolent SM & + & - & - & + \\
2 & Indolent SM & + & - & - & + \\
3 & Indolent SM & + & - & - & + \\
4 & Indolent SM & + & - & - & - \\
5 & Indolent SM & + & - & - & + \\
6 & Indolent SM & + & - & - & - \\
7 & Indolent SM & + & - & - & - \\
8 & Indolent SM & + & - & - & + \\
9 & Indolent SM & + & - & - & + \\
10 & Indolent SM & + & - & - & + \\
11 & Indolent SM & + & - & - & + \\
12 & Aggressive SM & - & - & - & + \\
13 & Aggressive SM & - & - & - & + \\
15 & Immunocytoma & + & + & - & - \\
16 & Immunocytoma & + & + & - & - \\
17 & Immunocytoma & + & + & - & - \\
\hline \multicolumn{2}{|l}{ SM, systemic mastocytosis. } & & & & \\
\hline
\end{tabular}

markers (CD5, CD20, CD23, CD25, $\kappa$ and $\lambda$ light chains, tryptase, chymase, and KIT). The diagnoses (table 1) comprised the following: indolent SM $(n=11)$, aggressive SM $(n=2)$, and lymphoplasmacytic immunocytoma $(n=3)$. Molecular biological studies were performed using a seminested polymerase chain reaction (PCR) technique for the demonstration of rearrangements of IgH and TCR $\gamma$. The DNA was extracted from the paraffin wax embedded sections according to standard protocols using phenol/chloroform/isoamyl alcohol (25/24/l, vol/vol/vol) after proteinase K digestion. TCR $\gamma$ and IgH PCRs were performed using protocols that have been described previously. ${ }^{67}$ Detection of the c-kit point mutation was performed using peptide nucleic acid mediated PCR clamping and Light Cycler hybridisation probes, also as described previously.

\section{RESULTS}

Histological findings in indolent SM were dominated by multifocal peritrabecular and/or perivascular infiltrates of densely packed MCs. A minor proportion of these compact MC infiltrates exhibited perifocal cohesive clusters of small lymphocytes without prominent cytomorphological atypia (fig lA). Although MC and lymphocytic infiltrates were often relatively sharply demarcated, atypical hypogranulated MCs could always be detected between the lymphocytes (fig lB). Aggressive SM also showed peritrabecular and perivascular compact MC infiltrates. In contrast to indolent SM, these were generally much larger, sometimes even confluent. However, lymphocytic aggregates were missing. Irrespective of the subtype, in almost all infiltrates a large proportion of MCs exhibited a spindle shaped appearance. The MCs strongly expressed KIT (CDI17) and tryptase (fig lC). Chymase expression was detected in almost all MCs in indolent SM, but was virtually absent in aggressive SM. Coexpression of CD25 was seen in almost all of the neoplastic MCs, whereas CD2 expression was inconstant and mostly weak or absent (fig 1D). Lymphocytic aggregates differed in size and shape. Sometimes, they formed a narrow rim around the MC infiltrates, but sometimes they were larger, occupying the central portions of the mixed MC/lymphocyte infiltrates. Cytomorphologically, the lymphocytes were mainly small to medium sized, with either round or irregularly shaped nuclei (fig 1B). Nucleoli were inconspicuous and intermingled blast cells were almost absent. Immunohistochemically, the clustered lymphocytes expressed B and T cell antigens (CD20 and $\mathrm{CD} 2 / \mathrm{CD} 5$, respectively) in nearly equal numbers (fig $\mathrm{IE}, \mathrm{F})$, whereas the diffuse lymphoid infiltrate of loosely scattered cells usually contained more T cells than B cells. An aberrant B cell immunophenotype with coexpression of CD20 and $\mathrm{CD} 5$ or $\mathrm{CD} 23$ was not detected in the investigated cases of SM and lymphoplasmacytic immunocytoma. Lymphoid infiltrates of immunocytoma were generally large, sometimes occupying whole bone marrow spaces, and showed sheets of CD20 positive, slightly pleomorphic, small to medium sized B
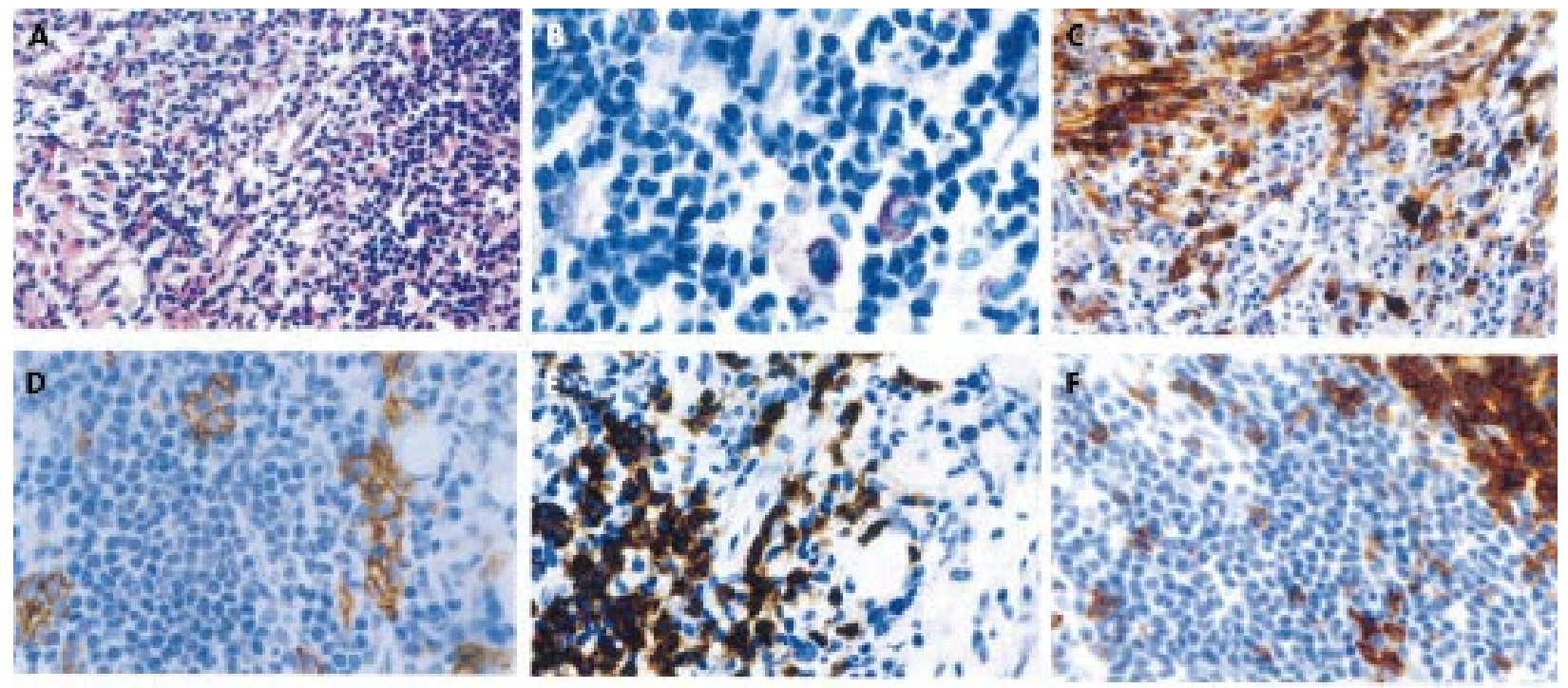

Figure 1 Bone marrow involvement in indolent systemic mastocytosis; a 55 year old female patient with adult-type urticaria pigmentosa. (A) A focal compact bone marrow infiltrate containing spindle shaped mast cells, intermingled eosinophils, and a large accumulation of lymphocytes; haematoxylin and eosin stain. (B) A higher magnification reveals sheets of small to medium sized lymphocytes, with slightly pleomorphic nuclei. Note a few loosely scattered hypogranulated mast cells. A diagnosis of mastocytosis should not be made on the basis of such findings alone; Giemsa stain. (C) Immunostaining with an antibody against tryptase is clearly positive for the mast cell infiltrates. Note that a large proportion of the mast cells has a clear spindle shaped appearance; avidin-biotin complex method (ABC); anti-tryptase antibody (AA 1). (D) Disseminated groups of mast cells with expression of CD25 can be easily detected within the large lymphoid infiltrate; $A B C$ method; anti-CD25 antibody. (E) A large proportion of the lymphocytes expresses the B cell associated antigen CD20; ABC method; anti-CD20 antibody (L26). (F) T cells expressing the antigen CD2 occur in nearly equal numbers to B cells (see (E)). Note that a large cluster of lymphocytes remains unstained; $A B C$ method; anti-CD2 antibody. 


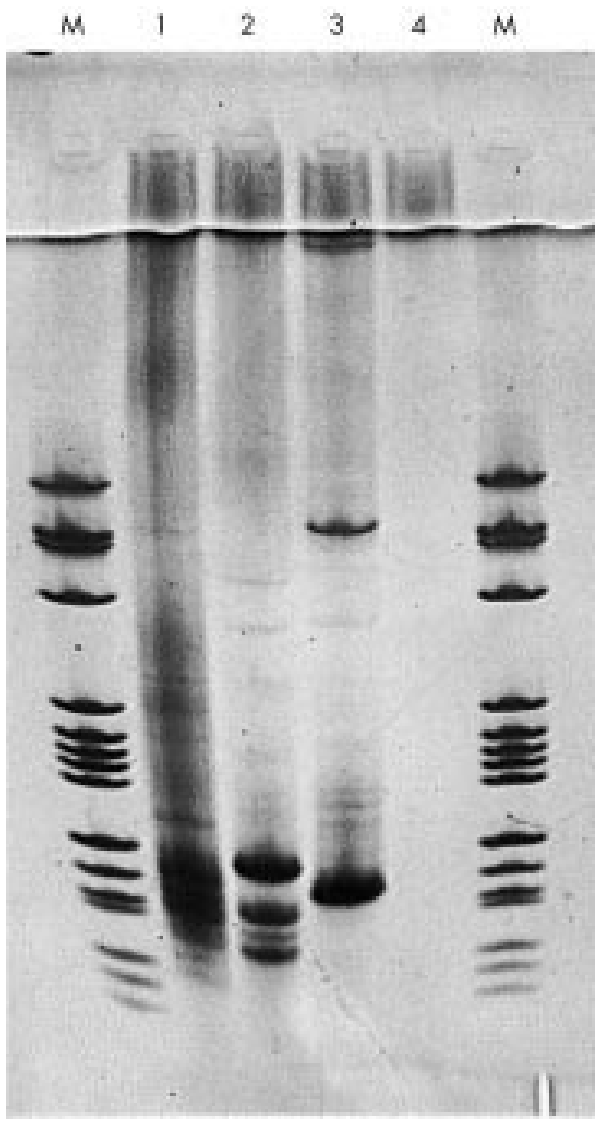

Figure 2 Representative results of polymerase chain reaction (PCR) analysis of heavy chain gene rearrangement. Silver stained 12\% polyacrylamide gel of PCR products from DNA samples obtained from patients with indolent systemic mastocytosis (lane 1), immunocytoma (lane 2), positive control (lane 3), and negative control (lane 4). M, low molecular weight marker. Whereas in indolent systemic mastocytosis the products show a polyclonal pattern, immunocytoma exhibits a monoclonal pattern.

cells. Clusters of blast cells were not detected. The intermingled plasma cells showed light chain restriction either for $\kappa$ or for $\lambda$, whereas plasma cells were polyclonal in all SM cases. The number of CD2 positive T cells was relatively low and did not exceed $20 \%$ of all lymphoid cells.

Molecular biological analyses revealed polyclonal rearrangements for IgH and TCR $\gamma$ in all cases of mastocytosis, but monoclonal rearrangement for IgH in all the three analysed cases of immunocytoma ( fig 2). However, T cells intermingled within the neoplastic B cell infiltrates showed a polyclonal rearrangement of the TCR $\gamma$. Point mutation Asp816-Val of the c-kit protooncogene was found in eight of 11 cases of indolent and all two cases of aggressive SM but was not detected in the three investigated cases of lymphoplasmacytic immunocytoma.

\section{DISCUSSION}

Lymphocytic aggregates are a relatively common histological finding in SM involving the bone marrow, in particular its indolent subvariant. ${ }^{9}{ }^{10}$ Immunohistochemistry has been used to analyse such aggregates, which were found to contain a mixture of B and T cells in nearly equal numbers (or with a slight preponderance of $\mathrm{T}$ cells), indicating a reactive process that could be termed "lymphocytosis". ${ }^{11}$ Vice versa, it is well known that a variety of reactive and neoplastic conditions affecting the bone marrow-for example, myelodysplasia, chronic lymphocytic leukaemia, hypocellular marrow states, especially cases of lymphoplasmacytic immunocytoma-can show a pronounced diffuse increase in usually loosely scattered MCs. ${ }^{45}$ Depending on the ratio of MCs to lymphocytes in a given section, the number of lymphocytes in indolent SM may exceed that of MCs by far, thus mimicking immunocytoma. In our present study, we tried to confirm the impression of a reactive increase in lymphocytes in SM by molecular biological techniques, and to establish a rationale for discriminating SM from immunocytoma on the basis of conventional histology, immunohistochemistry, and molecular biology.

Using conventional histology, the key feature for the diagnosis of SM with bone marrow involvement is the demonstration of at least one compact or dense infiltrate consisting of at least 10 to $15 \mathrm{MCs}$. Focal, compact MC infiltrates have been proposed to represent the one and only major criterion for the diagnosis of SM, according to the recently published new classification system. ${ }^{1}$ One of the four proposed minor diagnostic criteria for SM is the demonstration of an appreciable number of spindle shaped MCs within a given infiltrate. Immunocytoma may contain very high numbers of MCs, but these are always loosely scattered throughout the lymphomatous infiltrate, and never form compact infiltrates, as described before.

"The use of molecular biology clearly showed that local proliferations of B and T cells within the lymphocytic clusters of indolent systemic mastocytosis are reactive (benign) in nature, by demonstrating polyclonal rearrangements of $\mathrm{lgH}$ and TCR $\gamma^{\prime \prime}$

Using immunohistochemistry, it has been shown that the application of an antibody directed against the MC associated serine protease tryptase enables very quick screening and detection of even very small compact MC infiltrates. ${ }^{12}$ The estimation of MC numbers within the so called diffuse infiltrate, consisting of loosely scattered cells, which may be easily underestimated when only Giemsa or chloroacetate esterase stains are used, is also possible after the application of anti-tryptase antibodies. ${ }^{13}$ It has also been shown that an anti-CD25 antibody is a very sensitive marker for MCs (own unpublished observations, 2003). This finding is of particular diagnostic importance because previous flow cytometric studies have shown that CD25 expression occurs exclusively on neoplastic MCs, and the CD25 positivity of MCs has been included as a minor criterion in the list of clinicopathological criteria for establishing mastocytosis. ${ }^{14}$ Accordingly, we did not detect CD25 positive MCs in the three immunocytomas investigated (and also in a large number of control cases with MC hyperplasia; own unpublished observations, 2003). However, the use of antibodies against lymphocyte associated antigens seems to be of minor importance for the discrimination of SM from immunocytoma, particularly because the B cells of both disease entities express CD20 (but not CD5 or CD23), and therefore do not exhibit immunohistochemically detectable differences regarding their phenotypic features. It is of some importance that the proportion of admixed (reactive) $\mathrm{T}$ cells in the lymphocytic clusters of indolent SM was much higher ( $50 \%$ or more of all lesional lymphoid cells) than that seen in the infiltrates of the three cases of immunocytoma that we investigated ( $20 \%$ or less of all lesional lymphoid cells).

The use of molecular biology clearly showed that local proliferations of $\mathrm{B}$ and $\mathrm{T}$ cells within the lymphocytic clusters of indolent SM are reactive ("benign") in nature, by demonstrating polyclonal rearrangements of IgH and TCR $\gamma$, whereas there was a monoclonal proliferation of B cells, as expected, in all three cases of immunocytoma, accompanied by polyclonal $\mathrm{T}$ cells. The MCs of ten of the total of 13 analysed cases of SM were found to carry the typical point mutation (Asp-816-Val) of the c-kit protooncogene. Therefore, although polyclonal for $\mathrm{IgH}$ and TCR $\gamma$, it cannot be said with certainty that lesional 


\section{Take home messages}

- Immunohistochemical and molecular biological techniques showed that, with respect to the clonality of $\operatorname{lgH}$ and TCR $\gamma$ the perifocal lymphocytic clusters in bone marrow infiltrates of indolent systemic mastocytosis (SM) are reactive, and therefore can be regarded as lymphocytosis. A diagnosis of SM-AHNMD/non-Hodgkin lymphoma should not be made in such cases

- The application of antibodies against CD25 seems to be of superior value to delineate neoplastic from reactive mast cells

- It was also shown that the diagnostic criteria included in the new system of classification of mastocytosis need to be strictly applied in all suspected cases to assess or exclude mastocytosis on the basis of morphological and immunohistochemical findings in bone marrow biopsy specimens

lymphocytes do not belong to a neoplastic SM clone, because activating mutations of c-kit have been detected in the circulating lymphocytes of patients with SM and also in the neoplastic lymphoid cells of malignant T/natural killer cell lymphomas. ${ }^{15}{ }^{16}$ To clarify this issue further, the application of refined methods, in particular analysis of clonality in single microdissected pooled lymphocytes of a large number of cases, would be necessary.

\section{Authors' affiliations}

H-P Horny, K Lange, Institute of Pathology, University of Lübeck,

D-23538 Lübeck, Germany

K Sotlar, Institute of Pathology, University of Tübingen, D-72076

Tübingen, Germany

P Valent, University of Vienna, AKH, Department of Internal

Medicine/Hematology, A-1090 Vienna, Austria

\section{REFERENCES}

1 Valent P, Horny H-P, Escribano L, et al. Diagnostic criteria and classification of mastocytosis: a consensus proposal. Leuk Res 2001;25:603-25.
2 Metcalfe DD. Diagnosis and classification of mastocytosis: current status. J Invest Dermatol 1991;96:2S-4S.

3 Horny H-P, Kaiserling E. Lymphoid cells and tissue mast cells of bone marrow lesions in systemic mastocytosis: a histological and immunohistological study. Br J Haematol 1988;69:449-55.

4 Yoo D, Lessin LS, Jensen WN. Bone marrow mast cells in lymphoproliferative disorders. Ann Intern Med 1978;88:753-7.

5 Prokocimer M, Polliack A. Increased bone marrow mast cells in preleukemic syndromes, acute leukemia, and lymphoproliferative disorders. Am J Clin Pathol 1981;75:34-8.

6 Wright DK, Manos MM. Sample preparation from paraffin-embedded tissues. In: Innis AM, Gelfand DH, Sninsky JJ, et al, eds. PCR protocols: a guide to methods and applications. San Diego: Academic Press, 1990: 153-8.

7 Wang W, Kumar P, Schwarz M, et al. PCR amplification of 40 year-old paraffin-embedded tumour tissues: comparison of four different DNA extraction and purification methods. Int J Oncol 1994;5:453-7.

8 Sotlar K, Escribano L, Landt O, et al. One-step detection of c-kit point mutations using PNA-mediated PCR-clamping and hybridization probes. Am J Pathol 2003;162:737-46.

9 Horny H-P, Parwaresch MR, Lennert K. Bone marrow findings in systemic mastocytosis. Hum Pathol 1985;16:808-14.

10 Horny H-P, Valent P. Diagnosis of mastocytosis: general histopathological aspects, morphological criteria, and immunohistochemical findings. Leuk Res 2001;25:543-51.

11 Akin C, Jaffe ES, Raffeld M, et al. An immunohistochemical study of the bone marrow lesions of systemic mastocytosis: expression of stem cell factor by lesional mast cells. Am J Clin Pathol 2002;1 18:242-7.

12 Li WV, Kapadia SB, Sonmez-Alpan W, et al. Immunohistochemical characterization of mast cell disease in paraffin sections using tryptase, CD568, myeloperoxidase, lysozyme, and CD20 antibodies. Mod Pathol 1996;9:982-8

13 Horny H-P, Sillaber C, Menke D, et al. Diagnostic utility of staining for tryptase in patients with mastocytosis. Am J Surg Pathol 1998;22:1132-40

14 Escribano L, Orfao A, Villarrubia J, et al. Immunophenotypic characterization of human bone marrow mast cells. A flow cytometric study of normal and pathologic bone marrow samples. Anal Cell Pathol 1998; 16:151-9

15 Akin C, Kirshenbaum AS, Semere T, et al. Analysis of the surface expression of c-kit and occurrence of the c-kit Asp816Val activating mutation in T cells, B cells, and myelomonocytic cells in patients with mastocytosis. Exp Hematol 2000;28: 140-7.

16 Hongyo T, Li T, Syaifudin M, et al. Specific c-kit mutations in sinonasal natural killer/T cell lymphoma in China and Japan. Cancer Res 2000;60:2345-7. 